This item was submitted to Loughborough's Research Repository by the author.

Items in Figshare are protected by copyright, with all rights reserved, unless otherwise indicated.

\title{
Control strategy for a hand balance
}

PLEASE CITE THE PUBLISHED VERSION

PUBLISHER

(C) Human Kinetics

VERSION

VoR (Version of Record)

LICENCE

CC BY-NC-ND 4.0

REPOSITORY RECORD

Yeadon, Maurice R., and Grant Trewartha. 2019. "Control Strategy for a Hand Balance". figshare. https://hdl.handle.net/2134/6497. 
This item was submitted to Loughborough's Institutional Repository (https://dspace.lboro.ac.uk/) by the author and is made available under the following Creative Commons Licence conditions.

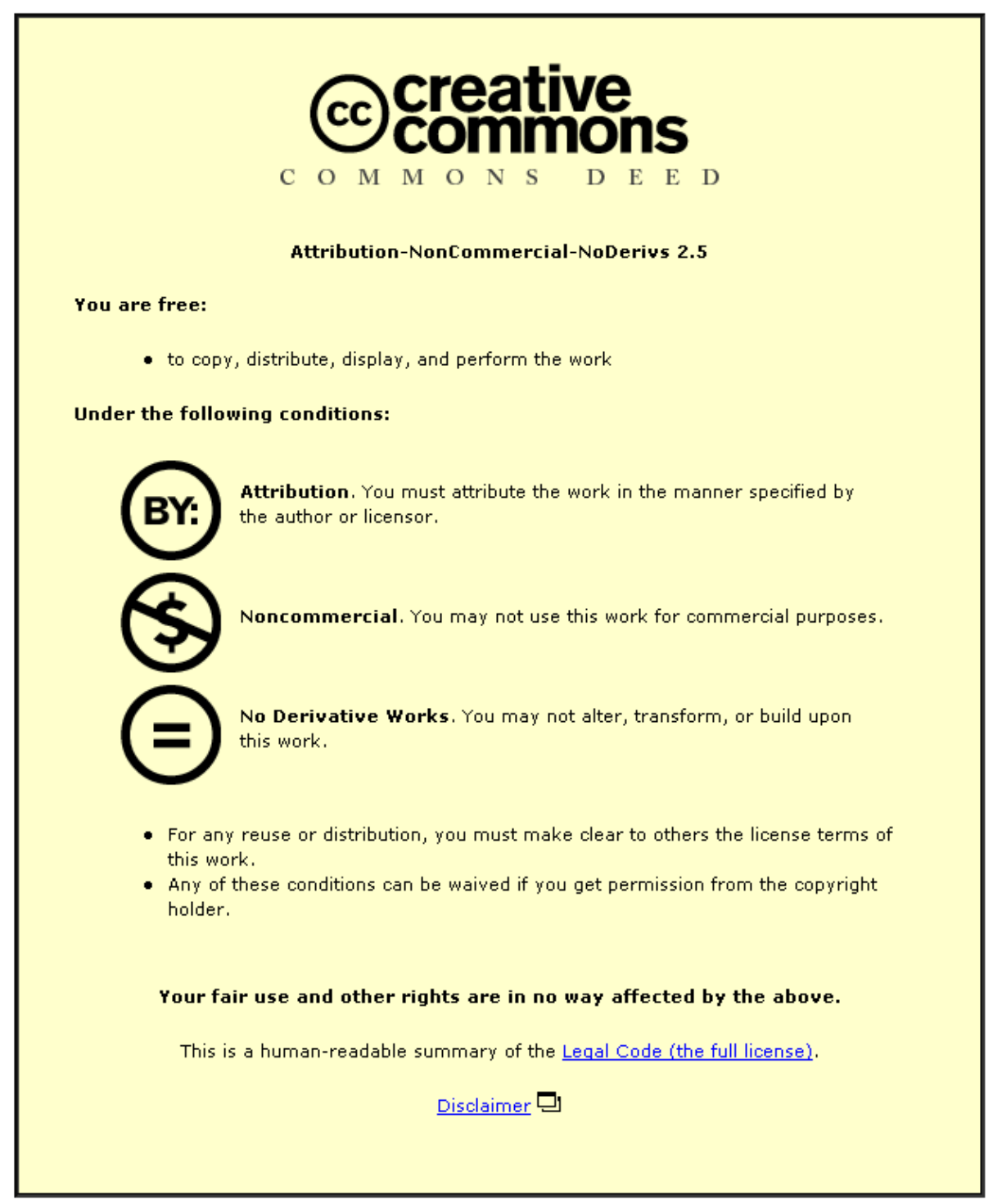

For the full text of this licence, please go to: http://creativecommons.org/licenses/by-nc-nd/2.5/ 


\title{
Control Strategy for a Hand Balance
}

\author{
Maurice R. Yeadon and Grant Trewartha
}

The goal of this study was to investigate the control strategy employed by gymnasts in maintaining a hand balance. It was hypothesized that a "wrist strategy" was used in which perturbations in the sagittal plane were corrected using variations in wrist flexor torque with synergistic shoulder and hip torques acting to preserve a fixed body configuration. A theoretical model of wrist strategy indicated that control could be effected using wrist torque that was a linear function of mass center displacement and velocity. Four male gymnasts executed hand balances and 2-dimensional inverse dynamics was used to determine net joint torque time histories at the wrist, shoulder, and hip joints in the sagittal plane. Wrist torque was regressed against mass center position and velocity values at progressively earlier times. It was found that all gymnasts used the wrist strategy, with time delays ranging from 160 to 240 $\mathrm{ms}$. The net joint torques at the shoulder and hip joints were regressed against the torques required to maintain a fixed configuration. This fixed configuration strategy accounted for $86 \%$ of the variance in the shoulder torque and $86 \%$ of the variance in the hip torque although the actual torques exceeded the predicted torques by $7 \%$ and $30 \%$, respectively. The estimated time delays are consistent with the use of long latency reflexes, whereas the role of vestibular and visual information in maintaining a hand balance is less certain.

Key Words: hand balance, handstand, inverted stance, control strategy

\section{Introduction}

From a link system perspective, a standing human comprises a mechanically unstable system. In order to maintain a "vertical orientation," the movement of the mass center must be controlled (Horak \& MacPherson, 1996). Horstmann and Dietz (1990) state that the mass center must remain vertically above the base of support in order to prevent falling, and while this requirement may not be necessary, it is certainly sufficient. This result is achieved by the generation of a series of muscular actions that work to produce torques about joints that maintain various body configurations and control the movement of the mass center (Hayes, 1988). These corrective torques arise from the visco-elastic nature of the muscle-tendon complexes crossing the joints (Horak \& MacPherson, 1996) and responses to information provided by muscle receptors, joint receptors, cutaneous

M.R. Yeadon is with the School of Sport and Exercise Sciences at Loughborough University, Loughborough, LE11 3TU, UK. G. Trewartha is with the Department of Sport \& Exercise Science, University of Bath, Bath BA2 7AY, UK. 
mechanoreceptors, vision, and the vestibular apparatus (Winter et al., 1990).

Two strategies have been identified in responses to perturbations of the support surface during stance. When the body sways forward in response to a backward movement of the supporting surface, the ankle strategy comprises a delayed activation of ankle extensors, knee flexors, and hip extensors, while the hip strategy consists of activation of the knee extensors and hip flexors (Horak \& Nashner, 1986). The two strategies may therefore be differentiated using the direction of hip torque. The ankle strategy is most useful for small slow perturbations, while the hip strategy is used for large or rapid perturbations or when the base of support is small, and little ankle torque is available (Horak \& Nashner, 1986).

The use of these two strategies for controlling quiet stance has been modeled using single joint link systems representing the human body (Camana et al., 1977; Hemami et al., 1978; Wu \& Zhao, 1997). More general models with three or four joints (Barin, 1989; He et al., 1991; Kuo, 1995) have been used to investigate control systems in which the controlling joint torques are linear functions of joint angles and angular velocities. The resulting matrices of gain coefficients are often difficult to interpret and are subject to wide variance. An interpretation of control strategy in terms of some simple criterion such as mass center behavior might provide more insight. Some models have incorporated representations of the vestibular apparatus (Camana et al., 1977; Hemami et al., 1978). The feedback time delays in these models range from zero to $400 \mathrm{~ms}$, although none has been used to determine the delay in experimental situations.

The time delays inherent in the various response possibilities are near zero for muscle visco-elasticity, 45-50 ms before the onset of EMG for myotatic reflexes (Nashner, 1976), 50-70 ms for tonic reflexes (Wu \& Zhao, 1997), 100-150 ms for long latency reflexes (Nashner, 1979), and 180-200 ms for visual and vestibular information (Nashner, 1976). In addition, there will be a further delay after the onset of EMG before the required joint torque is reached. Freund and Budingen (1978) found that the rise time of the fastest voluntary contractions of hand and forearm muscles was around $100 \mathrm{~ms}$ and was independent of the strength of the contraction and of the range of movement. The times for the force to rise successive thirds were 40,20 , and $40 \mathrm{~ms}$, and the rise time corresponding to maximum rate of rise was $55 \mathrm{~ms}$.

In a hand balance, the body is in an inverted posture and the equivalent of ankle and hip strategies will be wrist and hip (or shoulder) strategies. Since the aim of a hand balance in gymnastics is to remain motionless, it might be expected that a wrist strategy would be used in which the whole body remains in a near vertical fixed configuration. On the other hand, since the base of support is small, it may be necessary to use a hip strategy. When learning a hand balance, use is certainly made of vision, although this may be mainly to help calibrate the proprioceptive systems for a novel task (Gibson, 1958). In the learning stages, it is likely that vestibular input is also used, but the head is now located much lower in the body link system rather than at the end, as in normal stance. In a skilled hand balance, the relative use of these two feedback systems versus the faster reflex systems is not well-established.

It is hypothesized that a wrist strategy is used to maintain a hand balance and that the corrective torques at the shoulder and hip act synergistically in the same direction as the wrist torque. This study will use a single joint link model to formulate an expression for the controlling wrist torque in terms of mass center 
displacement and velocity and will investigate the extent to which this strategy describes the performance of gymnasts.

\section{Methods}

\section{Wrist Strategy}

Suppose that the body is modeled as a rigid segment of mass $m$ with mass center $G$ at a fixed distance $a$ from the fixed (wrist) axis $J$ connecting the hand and body segments (Figure 1). The aim of this control strategy is to maintain the horizontal displacement $x$ of the mass center $G$ close to a value $x_{0}$ using the (wrist) torque $T$ as controller so that $G$ remains vertically above the hand segment.

Taking moments about $J$ gives the equation of motion as:

$$
T-m g x=I \ddot{\phi}
$$

where $I$ is the moment of inertia about $J$.

If $\phi$ is close to $\pi / 2$, then $\ddot{x} \approx-a \ddot{\phi}$ so that:

$$
\left(T-m g x_{0}\right)-m g\left(x-x_{0}\right)=-\frac{I}{a} \ddot{x}
$$

Replacing $T_{1}=T-m g x_{0}$ and $x_{1}=x-x_{0}$ gives:

$$
\ddot{x}_{1}=k^{2} x_{1}-e T_{1} \text { where } k^{2}=\frac{m g a}{I} \text { and } e=\frac{a}{I}
$$

\section{Hip Strategy}

Suppose that the body is modeled as a planar two-segment system connected by the (hip) joint $J_{2}$ (Figure 2). The wrist strategy would require a wrist torque around $J_{1}$ tending to increase $\phi_{\mathrm{a}}$ together with a synergistic hip torque around $J_{2}$ tending to increase $\phi_{\mathrm{b}}$ or to resist any induced decrease in $\phi_{\mathrm{b}}$. In contrast the hip strategy uses a torque $T$ acting in the opposite direction (Figure 2). As a consequence, the direction of hip torque may be used as an indicator of which strategy is used.

\section{Control Strategy}

For the wrist strategy, the displacement $x_{1}$ of the system is governed by equation (2), which may be written as:

$$
\ddot{x}_{1}(t)=k^{2} x_{1}(t)-e T_{1}(t)
$$

where $t$ is the time.

If $T_{1}$ is based on the displacement $x_{1}$ and the velocity $\dot{x}_{1}$ at an earlier time due to the feedback time delay $t_{0}$ :

$$
T_{1}(t)=p x_{1}\left(t-t_{0}\right)+d \dot{x}_{1}\left(t-t_{0}\right)
$$


Using Taylor Series, $x_{1}\left(t-t_{0}\right)$ and $\dot{x}_{1}\left(t-t_{0}\right)$ may be approximated as:

$$
\begin{gathered}
x_{1}\left(t-t_{0}\right)=x_{1}(t)-t_{0} \dot{x}_{1}(t)+\frac{1}{2} t_{0}^{2} \ddot{x}_{1}(t) \\
\dot{x}_{1}\left(t-t_{0}\right)=\dot{x}_{1}(t)-t_{0} \ddot{x}_{1}(t)
\end{gathered}
$$

Substituting into (3):

$$
\left[1-\operatorname{det}_{0}+\frac{1}{2} p e t_{0}^{2}\right] \ddot{x}_{1}+e\left[d-p t_{0}\right] \dot{x}_{1}+\left[e p-k^{2}\right] x_{1}=0
$$

If each coefficient is positive, this is the equation for damped simple harmonic motion, and stable control will have been effected. The conditions for positive coefficients leads to:

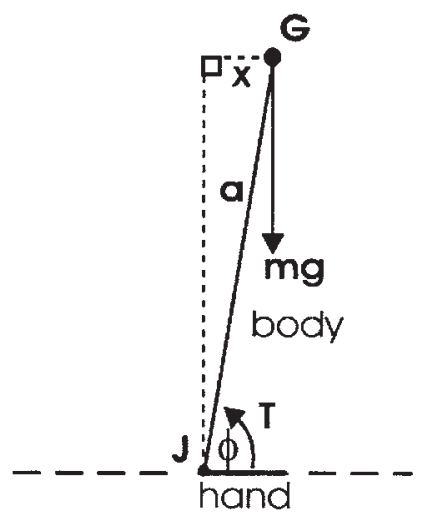

Figure 1 - A wrist joint model of hand balance control.

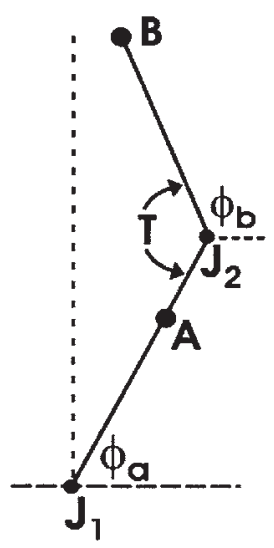

Figure 2 - A hip joint model of hand balance control. 


$$
\begin{gathered}
\frac{k^{2}}{e}<p<\frac{2}{e t_{0}{ }^{2}} \\
\frac{k^{2} t_{0}}{e}<d<\frac{2}{e t_{0}} \\
\frac{1}{2} k t_{0}<\frac{p}{d k}<\frac{1}{k t_{0}}
\end{gathered}
$$

Providing $t_{0}$ is not too large, there will be a range of values of $p$ and $d$ satisfying (8)-(10). For a given time delay $t_{0}$, the range of values of $p / d k$ that correspond to stable control is shown in Figure 3, which is based on (10). From (10) the limiting value of $t_{0}$ is $\sqrt{ } 2 / k$, with corresponding values of $p=k^{2} / e$ and $d=\sqrt{ } 2 k / e$. This approximate solution is in close agreement with that of Yeadon and Mikulcik (1996). For typical inertia values, the maximum time delay $t_{0}$ for wrist, shoulder, and hip strategies are each close to $0.5 \mathrm{~s}$.

\section{Data Collection}

In accordance with University Ethical Advisory Committee procedures, informed consent was obtained from 4 skilled male gymnasts (age $=16.5 \pm 2.9$ years, mass $=52.4 \pm 10.0 \mathrm{~kg}$, height $=1.61 \pm 0.07 \mathrm{~m}$, wrist-to-mass center distance $=0.90 \pm$ $0.06 \mathrm{~m}$ ) who performed handstands on a force platform. A calibration structure comprising six upright poles, each with five markers at vertical intervals of 0.5 $\mathrm{m}$, were positioned around a force plate (Kistler 9281-B12) forming a rectangular base of $1.025 \times 0.910 \mathrm{~m}$. Images of the calibration structure were recorded prior to the subject trials. Force and video data were recorded for each gymnast executing a series of handstand balances of $5 \mathrm{~s}$ in duration. Two genlocked video camera recorder systems (Sony Hi8, CCD and a Sony Hyper HAD 3CCD) were located on either side of the force plate, with the optical axis of each lens making an angle of $45^{\circ}$ with the sagittal plane. The cameras operated at 50 fields per second with electronic shutters set to $1 / 215 \mathrm{~s}$ and $1 / 250 \mathrm{~s}$, respectively. The force plate data were sampled via an analog-to-digital converter (CED1401) at $200 \mathrm{~Hz}$. Force capture was triggered manually during a hand balance, and sequential illumination of an LED unit at 1-ms intervals permitted synchronization of the two sets of video data with the force data. Markers were positioned on the posterior surface of the body at points corresponding to joint centers. Personalized body segment inertia parameters were generated from anthropometric measurements using the inertia model of Yeadon (1990b), which also included a mass ratio correction factor to ensure that calculated body mass was identical to body mass obtained from weighing.

\section{Data Processing}

Video images of the calibration structure were manually digitized for each camera view. Camera calibration was effected using an 11 parameter Direct Linear Transformation procedure (Abdel-Aziz \& Karara, 1971), and unbiased estimates 
of reconstruction accuracy were determined. In each field of the two views of a hand balance, the images of the surface markers on the body were digitized, and three-dimensional coordinates were reconstructed along with reliability estimates. The offsets between markers and joint centers obtained from physical measurements were then used to obtain the coordinates of wrist, elbow, shoulder, hip, knee, and ankle joint centers together with finger, toe, and head center locations. Quintic splines (Wood \& Jennings, 1979) were fitted to the coordinates to obtain interpolated values. By observing the number of illuminated LEDs in the unique initial video field, the time offset from the start of the force capture was determined, and matched force and interpolated video data were output at $50 \mathrm{~Hz}$.

Joint center coordinates and segmental inertia parameters were used to obtain wrist, shoulder, and hip angles $\phi_{\mathrm{a}}, \phi_{\mathrm{b}}, \phi_{\mathrm{c}}$ (Yeadon, 1990a), and segmental and whole-body mass center coordinates (Figure 4). A pseudo coordinate data set was obtained from the original coordinate data set by replacing each data point by the point representing the average between the preceding data point and proceeding data point. The difference values arising from these two data sets were used to estimate the precision of $\phi_{\mathrm{a}}, \phi_{\mathrm{b}}, \phi_{\mathrm{c}}$, and the whole-body mass center displacement. Quintic splines were fitted to $\phi_{a}, \phi_{b}, \phi_{c}$, and first and second derivatives were calculated.

The double integral of fore-aft horizontal acceleration, obtained by dividing the horizontal force $F_{\mathrm{x}}$ by body mass, was subtracted from video-obtained wholebody mass center position values. The resulting quantity was regressed against time and time squared to determine equation constants for initial position $x_{0}$, initial velocity $v_{0}$, and an error term $e$. Reevaluated mass center position values were determined using the following equation:

$$
x=\iint\left(F_{x} / m\right) d t+x_{0}+v_{0} t+e t^{2}
$$

Furthermore, mass center velocity values were recalculated using the following equation:

$$
v=\int\left(F_{x} / m\right) d t+v_{0}+2 e t
$$

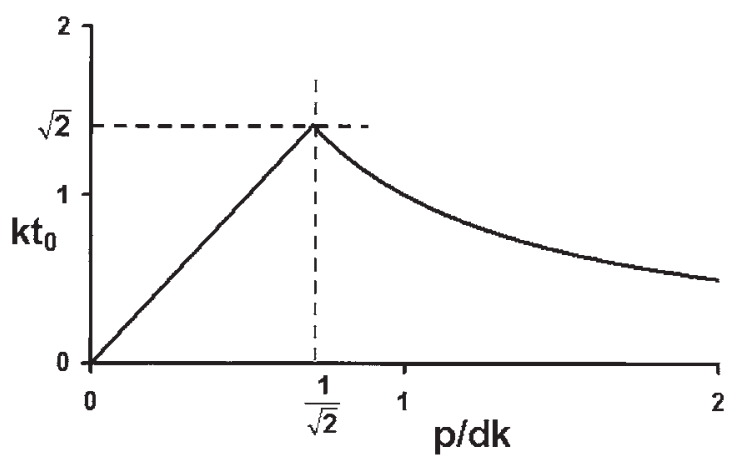

Figure 3 - The area under the graph corresponds to $p, d$, and $t_{0}$ values for which there is stable control. 
The reevaluated displacements were derived from force data and thus considered to be more accurate than manually digitized displacement data estimates and their derivatives; the inclusion of the $t^{2}$ term corrected for systematic force plate error. An additional $(0.0040 \pm 0.0026 \mathrm{~m})$ systematic correction was applied to the body mass center values by minimizing the RMS difference between mass center and center of pressure position data so that mean values were identical (Gurfinkel et al., 1992). The precision of the new mass center displacement values was estimated in the same way as that of the video values using a pseudo data set.

\section{Inverse Dynamics}

Coordinate, force, and inertia data were combined to conduct two-dimensional inverse dynamics analysis. Each gymnast was represented by a four-segment model (Figure 4), consisting of a hand, arm, trunk+head, and leg segment, with three corresponding joints: wrist, shoulder, and hip.

For each of the four segments, equations of motion were developed giving three equations for each segment and 12 equations in total. Eliminating reactions at each of the three joints left six equations to be solved for six unknowns: $T_{1}$,

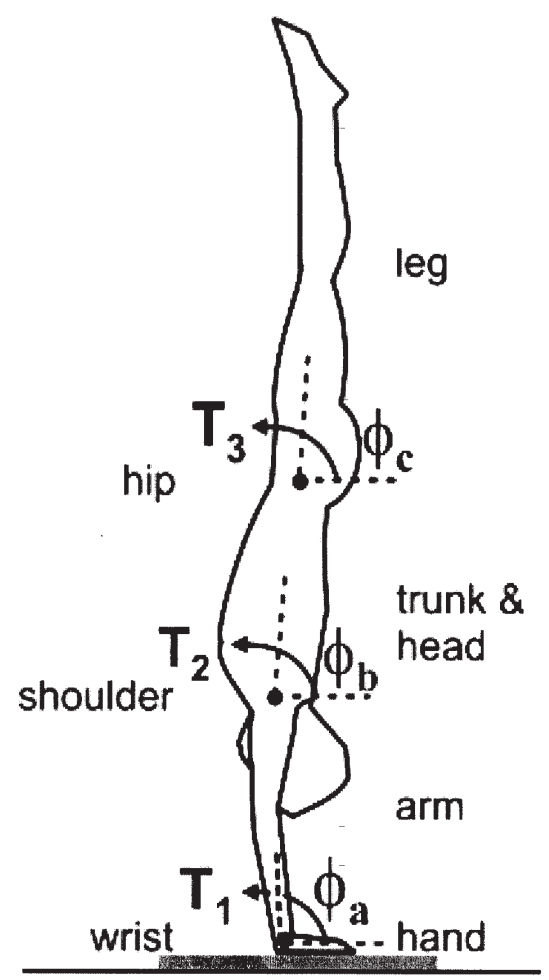

Figure 4 - Four-segment representation of gymnast in hand balance. 
$T_{2}, T_{3}, \ddot{\phi}_{\mathrm{a}}, \ddot{\phi}_{\mathrm{b}}, \ddot{\phi}_{\mathrm{c}}$ (see Appendix). Estimated values from splines for the angular accelerations were included as three further equations, giving nine equations that were solved in a linear, least-squares manner for three joint torques (and three angular accelerations) while preventing the occurrence of outlying torque values evident in the solving of the six equations (Figure 5).

\section{Data Analysis}

This study aimed to investigate the coordinated feedback strategy used to maintain balance in a handstand. In order to remove the confounding effects of high frequency vibrations due to muscle stiffness (Bach et al., 1983) and low frequency drift (Zatsiorsky \& Duarte, 2000), the data were filtered. The resonance frequency due to the viscoelasticity of the ankle extensors is around $3 \mathrm{~Hz}$ (Bach et al., 1983) while, in quiet stance, more than $90 \%$ of the power spectrum is below $2 \mathrm{~Hz}$, with peak power around $0.4 \mathrm{~Hz}$ (Soames \& Atha, 1982), and the low frequency component is below $0.2 \mathrm{~Hz}$ (Winter et al., 1996). In the present study on hand balances, the mean frequency at which peak power occurred was $0.6 \mathrm{~Hz}$, with $96 \%$ of the total power below $2 \mathrm{~Hz}$. The calculated joint torques, mass center position, and velocity and angle derivative data sets were each linearly detrended using a least-squares line and fitted with a Fourier cosine series. These cosine series were filtered (truncated) below $0.2 \mathrm{~Hz}$ and above $2 \mathrm{~Hz}$. The detrending also removed the systematic offsets arising from the mass center lying above the hand rather than the wrist center so that the detrended data oscillated about zero (Figures $6 \& 7$ ).

Multiple regressions of wrist torque $T_{1}(t)$ against mass center displacement $x\left(t-t_{0}\right)$ and velocity $\dot{x}\left(t-t_{0}\right)$ were conducted on all trials for time delays varying from 0 to $1 \mathrm{~s}$ taken at $0.02-\mathrm{s}$ increments in order to determine the time delays corresponding to maximum $R^{2}$. The shoulder and hip torques $T_{2}$ and $T_{3}$ were each regressed against $T_{1}$ to verify that they acted synergistically with $T_{1}$ and to investigate the hypothesis that the relationships between torques are fixed (Yang et al., 1990).

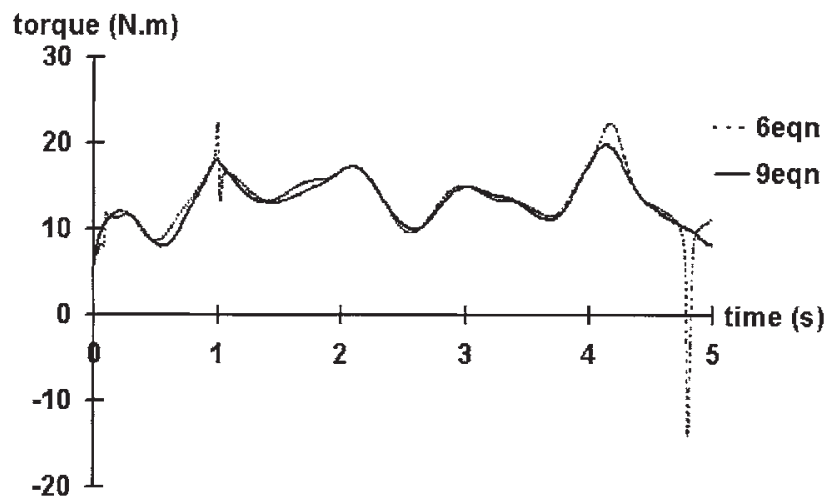

Figure 5 - Outlying torque values when solving from six equations. 
The theoretical torques $T_{2}^{\prime}$ and $T_{3}^{\prime}$ required at the shoulder and hip joints for maintaining a fixed body configuration in order to support control at the wrist were determined, detrended, and filtered. $T_{2}$ ' was calculated with the assumption that the hip joint was fixed, whereas $T_{3}$ ' was calculated using the actual motion at the shoulder joint. It should be noted that there is no ideal method of estimating the torques that would preserve configuration during a given movement if configuration changed in the actual movement. The actual torques $T_{2}$ and $T_{3}$ were then regressed against $T_{2}^{\prime}$ and $T_{3}^{\prime}$ at earlier times to establish the strength of the relationships and to determine any time delay between $T_{1}$ and $T_{2}$, and $T_{1}$ and $T_{3}$ involved in holding a fixed body configuration.

All regression analyses were also carried out with data that were detrended but not filtered above $2 \mathrm{~Hz}$.

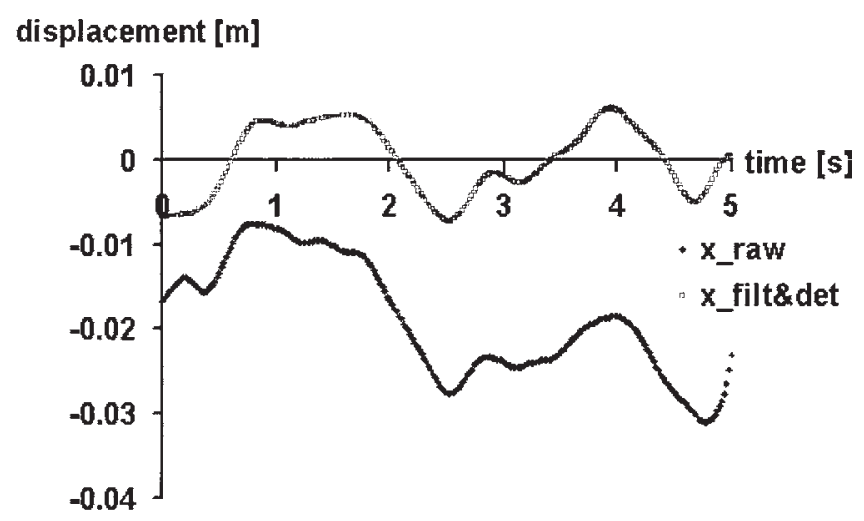

Figure 6 - Time histories of anterior-posterior mass center displacement for one example subject, raw data (x_raw) and data after filtering and detrending (x_filt\&det).

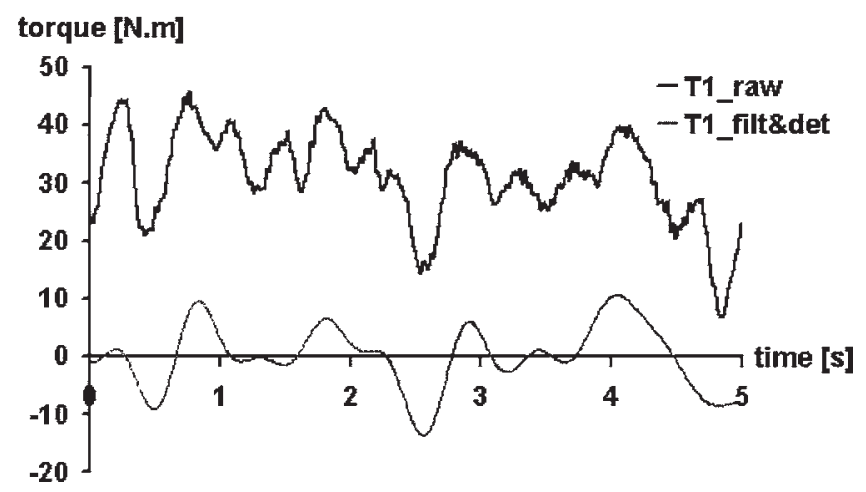

Figure 7 - Time histories of wrist torque for one subject, including raw data (T1_raw) and data after filtering and detrending (T1_filt\&det). 


\section{Results}

The reconstruction errors of the calibration markers were $0.0033 \mathrm{~m}, 0.0027 \mathrm{~m}$, and $0.0022 \mathrm{~m}$ in the anterior-posterior, medio-lateral and vertical directions, respectively. Average reliability estimates of the three-dimensional reconstruction of body landmarks ranged from 0.008 to $0.009 \mathrm{~m}$. Precision estimates of the mass center location (obtained from digitization) ranged from 0.0016 to $0.0020 \mathrm{~m}$, and precision estimates of angle values ranged from 0.9 to $1.2^{\circ}$ across the four trials. Precision estimates of the mass center location (obtained from double integration of the force plate data) ranged from 0.00004 to $0.00008 \mathrm{~m}$.

The wrist torque $T_{1}$ was significantly $(p<.001)$ correlated with the mass center displacement and velocity for each subject with estimated time delays ranging from 160 to $240 \mathrm{~ms}$ (Table 1). When unfiltered data were used, the time delays ranged from 160 to $180 \mathrm{~ms}$ (Table 2). The coefficient values can be compared with the theoretical values corresponding to maximum controllable delay time and the expected coefficient ranges for the estimated time delays obtained using inequalities (8), (9), and (10) (Table 3).

Table 1 Regressions of Wrist torque $T_{1}(t)$ Against Mass Center Displacement $x(t$ $\left.-t_{0}\right)$ and Velocity $\dot{x}\left(t-t_{0}\right)$ for $t_{0}$ That Maximises $R^{2}$

\begin{tabular}{lcccc}
\hline Subject & $R^{2}$ & Delay $(\mathrm{ms})$ & $x$ coef & $\dot{x}$ coef \\
\hline A & 0.76 & 240 & 785 & 280 \\
B & 0.74 & 200 & 644 & 255 \\
C & 0.86 & 220 & 875 & 232 \\
D & 0.75 & 160 & 870 & 107 \\
\hline
\end{tabular}

Note. All regressions were significant at the 0.001 level. All data filtered above $2 \mathrm{~Hz}$.

Table 2 Regressions of Wrist Torque $T_{1}(t)$ Against Mass Center Displacement and Velocity $\dot{x}\left(t-t_{0}\right)$ for $t_{0}$ That Maximizes $R^{2}$

\begin{tabular}{lcccc}
\hline Subject & $R^{2}$ & Delay $(\mathrm{ms})$ & $x$ coef & $\dot{x}$ coef \\
\hline A & 0.50 & 160 & 815 & 241 \\
B & 0.56 & 160 & 549 & 207 \\
C & 0.73 & 180 & 1006 & 195 \\
D & 0.56 & 160 & 596 & 145 \\
\hline
\end{tabular}

Note. All regressions were significant at the .001 level. All data unfiltered. 
Table 3 Actual and Limiting Time Delays and the Corresponding Coefficients for Each Subject

\begin{tabular}{|c|c|c|c|c|c|c|c|c|}
\hline \multirow[t]{2}{*}{ Subject } & \multicolumn{2}{|c|}{ Delay (ms) } & \multicolumn{3}{|c|}{$x$ coefficient } & \multicolumn{3}{|c|}{$\dot{x}$ coefficient } \\
\hline & Actual & Limit & Min & $\operatorname{Max}$ & Limit & Min & $\operatorname{Max}$ & Limit \\
\hline A & 240 & 491 & 541 & 2270 & 541 & 130 & 545 & 266 \\
\hline B & 200 & 497 & 506 & 3120 & 506 & 101 & 622 & 251 \\
\hline C & 220 & 475 & 609 & 2835 & 609 & 134 & 624 & 289 \\
\hline D & 160 & 468 & 374 & 3198 & 374 & 60 & 512 & 175 \\
\hline
\end{tabular}

Figure 8 shows the results of regressions carried out between wrist torque and delayed mass center position, and velocity values for one typical trial. For each wrist torque, the largest amount of variance accounted for by $x$ and $\dot{x}$ in a combined regression occurs at the estimated time delay. Univariate regressions of $T_{1}$ against $x$ and $T_{1}$ against $\dot{x}$ are also shown. Note that at zero delay, $T_{1}$ is negatively correlated with $\dot{x}$.

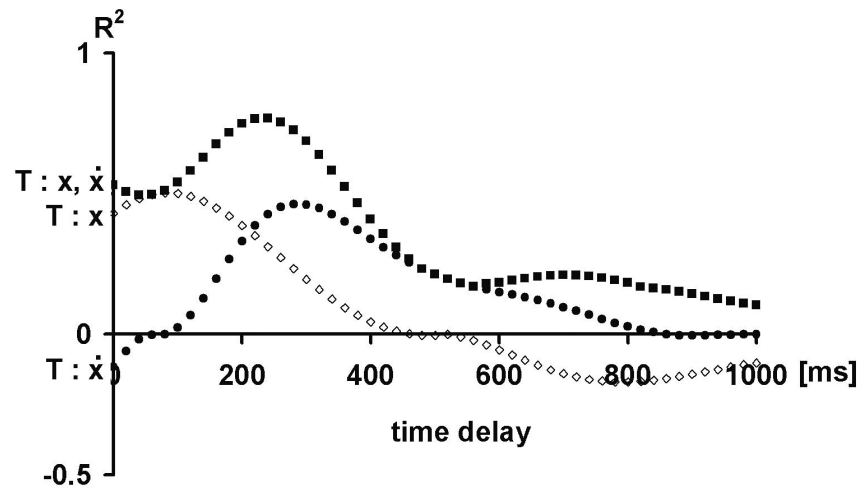

Figure $8-$ Regressions of wrist torque $T_{1}(t)$ against displacement $x\left(t-t_{0}\right)$ and velocity $\dot{x}\left(t-t_{0}\right)$ for $0<t_{0}<1 \mathrm{~s}$.

The regressions of $T_{2}$ and $T_{3}$ against $T_{1}$ had positive coefficients (Tables $4 \& 5$ ), showing that the directions of the shoulder and hip torques were the same as that of the wrist torque, consistent with the use of the wrist strategy. The regressions of $T_{2}$ against the torque $T_{2}$ ' required to maintain a fixed configuration show a marginally stronger relationship (Table 4). The estimated time delay between $T_{2}$ and $T_{2}$ ' was zero or close to zero in each case. The regressions of $T_{3}$ against the torque $T_{3}$, required to maintain a fixed configuration show a stronger relationship than between $T_{3}$ and $T_{1}$ (Table 5). The estimated time delays between $T_{3}$ and $T_{3}$ ' ranged from 20 to 80 $\mathrm{ms}$ for filtered data but were zero for unfiltered data. 


\section{Discussion}

The strategy used by each gymnast was a compensatory wrist torque to control the mass center displacement and velocity together with synergistic torques at the shoulder and hip generally acting in the same direction as the wrist torque. This was consistent with the results of Kerwin and Trewartha (2001) who found that wrist, shoulder, and hip torques were significantly correlated with mass center displacement, with wrist torque predominant. Another technique involving elbow flexion was evident in Slobonov and Newell (1996) and was probably adopted after a failure of wrist strategy to maintain balance. This is reflected in the mean range of $0.50 \mathrm{~m}$ in ankle displacement compared with $0.12 \mathrm{~m}$ in the present study.

The mathematical formulation of the wrist strategy based on a simple twosegment model comprising the hand and remainder of the body was consistent with the statistical relationship between the wrist torque data expressed as a linear function of mass center displacement and velocity (Table 1). This relationship explained more than $74 \%$ of the variance in the wrist torque and gave regression coefficients comparable with those expected from theoretical considerations (Table $3)$. While it is encouraging to have such a simple description of the overall strategy, there is still the unexplained variance to be accounted for. This may be a result of noise in the feedback information leading to errors in response, or it may indicate that the strategy used is somewhat different.

It is evident that the synergistic torques at the shoulder and hip were more complex than a simple proportion of the wrist torque although there was quite a strong correlation between shoulder torque and wrist torque $\left(R^{2}>0.76\right)$ and a weaker relationship $\left(R^{2}<0.64\right)$ between hip torque and wrist torque (Tables $\left.4 \& 5\right)$. The shoulder torque required to maintain a fixed-body configuration was slightly more strongly correlated $\left(R^{2}>0.77\right)$ with the actual torque and underestimated the actual torque by only $6 \%$ on average. The fixed configuration strategy explained more of the hip torque variance $\left(R^{2}>0.58\right)$ than a fixed proportion of wrist torque but underestimated the hip torque by $23 \%$ on average (Table 5). Whether this is a consequence of a different strategy being used is not clear.

Estimates of the delay in the feedback system ranged from 160 to $240 \mathrm{~ms}$ (Table 1). This delay includes the time for the torque to rise or fall to the required level once the change in enervation signal has arrived at the muscle motor units. Since the wrist flexor torque is always active in a hand balance (Figure 7), the time required to rise to a new level will be smaller than the $100 \mathrm{~ms}$ (Freund \& Budingen, 1978) required to ramp up from zero, say around $40 \mathrm{~ms}$. The latency in the response of the enervation to the wrist flexor muscles will therefore range from around 120 to $200 \mathrm{~ms}$. Since visual and vestibular latencies are greater than $180 \mathrm{~ms}$ (Nashner, 1976), a time delay of $120 \mathrm{~ms}$ suggests that visual and vestibular feedback were not used, whereas a delay of $200 \mathrm{~ms}$ suggests that visual or vestibular feedback was used. There is also the possibility that control based on visual and vestibular feedback augments the control based on proprioceptive feedback with long latency (100-150 ms), and the estimated time delays reflect the two delay times.

On the other hand, visual and vestibular feedback may be used to control the longer term stability of the system (Collins \& De Luca, 1995a) and to correct for drift in the short delay controller. Since it is possible for an elite gymnast to perform hand balances with eyes closed, it may be that vision is only important for the short term controller in the learning stages (Gibson, 1958). 

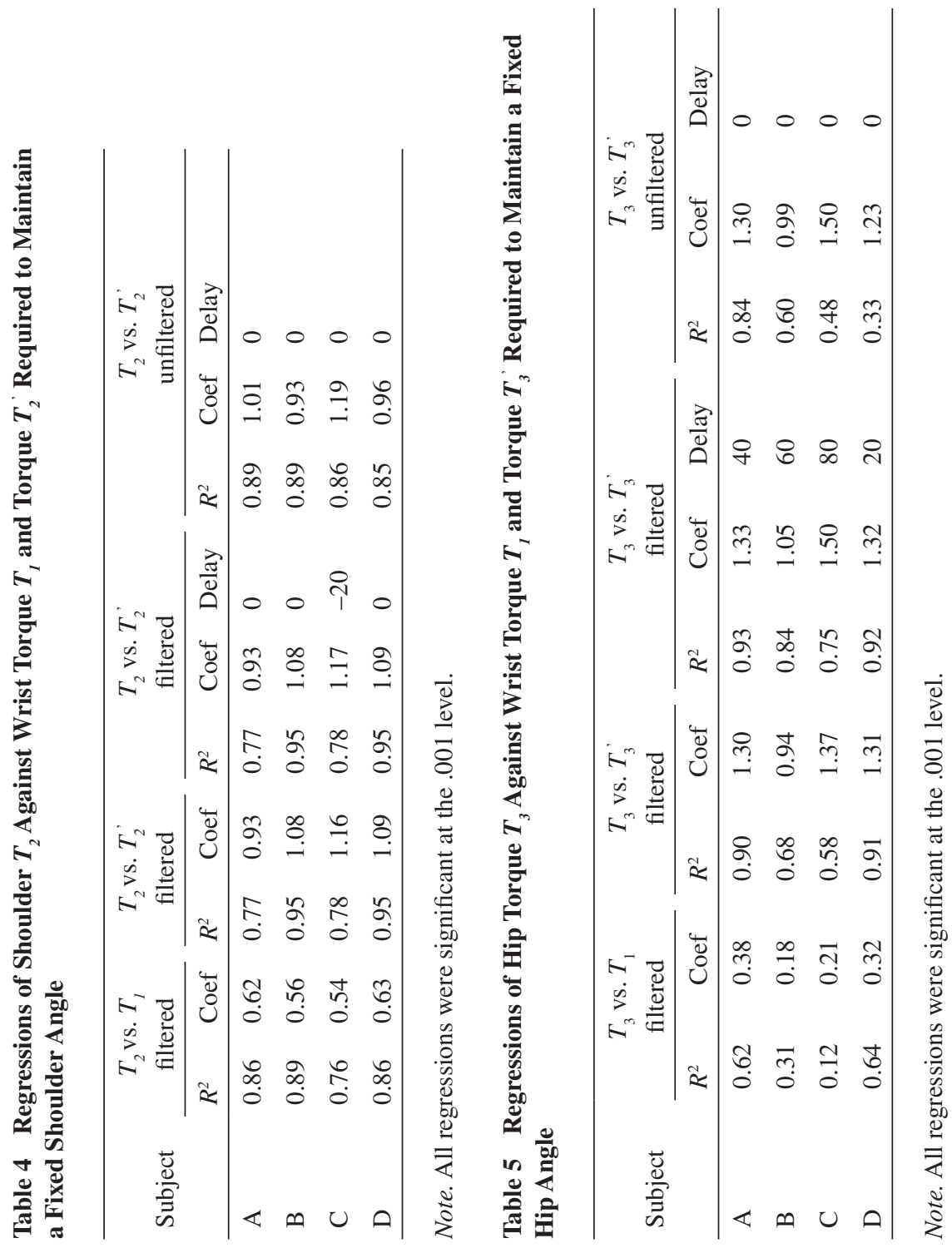
The linear relationship between wrist torque and mass center displacement and velocity was still evident when unfiltered data was used (Table 2), although the correlations were smaller $\left(0.50<R^{2}<0.73\right)$ as might be expected, since the high frequency components will appear mainly as noise. The estimated time delays were $40 \mathrm{~ms}$ shorter on average than those obtained from the data filtered above 2 Hz. This might be expected, since the inclusion of the muscle stiffness response, which has a delay close to zero, will affect the overall estimated delay in this simplified model. There was little difference in the relationship between the actual shoulder torque and that required to maintain a fixed configuration when unfiltered data was used (Table 4). In the case of hip torque, the filtered data indicated an additional delay of around $50 \mathrm{~ms}$ relative to the wrist torque, while the unfiltered data had no such additional delay but exhibited lower correlations (Table 5). Again the response of muscle stiffness would be expected to reduce any such delay in the case of unfiltered data. Any delay in enervation of the hip extensors or flexors might be expected to be less than this $50 \mathrm{~ms}$ mean value, since the rise times of these muscles are greater than the $100 \mathrm{~ms}$ for the wrist flexors (Bobbert \& Zandwijk, 1999). However, the same tendency to have longer delays outwards along the link system has been observed in perturbations of normal stance, where the muscle latencies at the hip were, on average, around $27 \mathrm{~ms}$ greater than those at the knee, which in turn were some $20 \mathrm{~ms}$ greater than those at the ankle (Horak \& Nashner, 1986). The synergistic hip torque, which aims to preserve the hip angle, will be augmented by passive zero delay joint stiffness if the correct torque level is not generated. Additionally, it is conceivable that myotatic (short latency) reflexes also operate to help stabilize the joint and that the observed delays are a consequence of having a synergistic hip torque that is synchronous with the wrist torque and is augmented by a short latency reflex response.

This study has provided estimates of the feedback time delay in the control of hand balances. Previously, latencies have only been obtained for responses to perturbations of stance rather than for the control of quiet stance. Since more than one mechanism is used to effect overall control, it will be illuminating if the various contributions can be differentiated. Eklund and Löfstedt (1970) filtered ankle torque at different frequencies to separate out the information content of the signal. Collins and De Luca (1995b) used stabilogram-diffusion analysis to identify both short-term $(<1 \mathrm{~s})$ and long-term $(>1 \mathrm{~s})$ control schemes operating in quiet stance. Schumann et al. (1995) used a nonstationary spectral estimation technique to identify changes in the postural control system over time. Fitzpatrick et al. (1992) used cross-correlation and frequency analysis to demonstrate that much of the reflex response originates from lower limb mechanoreceptors stimulated by ankle rotation. Techniques of this type are needed to identify the roles of the different control mechanisms and their latencies.

While many torque-driven models of balance control have assumed zero response latencies (Barin, 1989; Camana et al., 1977; Hemami \& Katbab, 1982) and a few have used non-zero latencies-30 ms (Wu \& Zhao, 1997), $185 \mathrm{~ms}$ (Hemami et al., 1978) — it appears that only Wu and Zhao (1997) seem to have included an additional delay $(40 \mathrm{~ms})$ for the torque to rise or fall to a new level. This problem could be overcome by incorporating muscle models with activation dynamics into a link system model (He et al., 1991).

The mass center displacement and velocity values used by the wrist strategy may be obtained as linear functions of joint angles and angular velocities, which in 
turn are provided by joint and muscle receptors. Additionally, mass center velocity might be provided by cutaneous receptors in the hands, since the vertical component of the ground reaction force should vary with the velocity squared (Horstmann \& Dietz, 1990; Wu \& Zhao, 1997).

Elite gymnasts have little difficulty in maintaining a hand balance with eyes closed but find greater difficulty if the neck is flexed reorienting the head and vestibular apparatus. The latter effect may be a consequence of placing the vestibular apparatus in an unfamiliar orientation or may suggest that some use is made of vestibular information in maintaining control. On the other hand, Fitzpatrick and McCloskey (1994) found that the vestibular system played no part in the perception of sway during normal standing.

While much is now known about the control of balance, it will continue to be a challenging area for research due to the complexity of the sensory system and the sensitivity of the adjustments made. Hand balances by elite gymnasts provide an alternative to normal stance for studying such control. The hand balance task differs in that symmetry, extension, and stillness are required (akin to standing to attention), whereas upright stance is generally not so tightly prescribed, the mass distribution and geometry as well as the location and orientation of the vestibular and visual apparatus within the link system are quite different, and the high tactile resolution of the hand may also be an important difference. Nevertheless the two tasks share common processes such as the estimation of the spatial arrangement and motion of the body, and the implementation of a multi-joint strategy to preserve orientation and configuration within certain constraints. As a consequence, an understanding of mechanisms or strategies in one task may lead to insights into the other.

\section{References}

Abdel-Aziz, Y.I., \& Karara, H.M. (1971). Direct linear transformation from comparator coordinates into object-space coordinates. In Close range photogrammetry (pp.1-18). Falls Church, VA: American Society of Photogrammetry.

Bach, T.M., Chapman, A.E., \& Calvert, T.W. (1983). Mechanical resonance of the human body during voluntary oscillations about the ankle joint. Journal of Biomechanics, $16,85-90$.

Barin, K. (1989). Evaluation of a generalized model of human postural dynamics and control in the sagittal plane. Biological Cybernetics, 61, 37-50.

Bobbert, M.F., \& van Zandwijk, J.P. (1999). Dynamics of force and muscle stimulation in human vertical jumping. Medicine and Science in Sports and Exercise, 31, 303310.

Camana, P.C., Hemami, H., \& Stockwell, C.W. (1977). Determination of feedback for human posture control without physical intervention. Journal of Cybernetics, 7, 199-225.

Collins, J.J., \& De Luca, C.J. (1995a). The effects of visual input on open-loop and closedloop postural control mechanisms. Experimental Brain Research, 103, 151-163.

Collins, J.J., \& De Luca, C.J. (1995b). Upright, correlated random walks: A statisticalbiomechanics approach to the human postural control system. Chaos, 5, 57-63.

Eklund, G., \& Löfstedt, L. (1970). Bio-mechanical analysis of balance. Biomedical Engineering, 5, 333-337.

Fitzpatrick, R., \& McCloskey, D.I. (1994). Proprioceptive, visual and vestibular thresholds for the perception of sway during standing in humans. Journal of Physiology, 478, 173-186. 
Fitzpatrick, R.C., Gorman, R.B., Burke, D., \& Gandevia, S.C. (1992). Postural proprioceptive reflexes in standing human subjects: bandwidth of response and transmission characteristics. Journal of Physiology, 458, 69-83.

Freund, H.J., \& Budingen, H.J. (1978). The relationship between speed and amplitude of the fastest voluntary contractions of human arm muscles. Experimental Brain Research, 31, 1-12.

Gibson, J.J. (1958). Visually controlled locomotion and visual orientation in animals. British Journal of Psychology, 493, 182-194.

Gurfinkel, V.S., Popov, K.E., \& Smetanin, B.N. (1992). The support input as a reference for postural control. In M. Woollacott \& F. Horak (Eds.), Posture and gait: Control mechanisms (pp.186-189). Eugene, OR: University of Oregon Books.

Hayes, K.C. (1988). Biomechanics of postural control. Exercise \& Sport Sciences Review, 10, 363-391.

He, J., Levine, W.S., \& Loeb, G.E. (1991). Feedback gains for correcting small perturbations to standing posture. IEEE Transactions on Automatic Control, 36, 322-332.

Hemami, H., \& Katbab, A. (1982). Constrained inverted pendulum model for evaluating upright postural stability. Journal of Dynamic Systems, Measurement, and Control, 104, 343-349.

Hemami, H., Weimer, F.C., Robinson, C.S., Stockwell, C.W., \& Cvetkovic, V.S. (1978). Biped stability considerations with vestibular models. IEEE Transactions on Automatic Control, AC-23, 1074-1079.

Horak, F.B., \& MacPherson, J.M. (1996). Postural orientation and equilibrium. In L.B. Rowell \& J.T. Shepherd (Eds.), Handbook of physiology. Section 12: Exercise: Regulation and integration of multiple systems (pp. 255-292). Oxford University Press.

Horak, F.B., \& Nashner, L.M. (1986). Central program of postural movements: Adaptations to altered support-surface configurations. Journal of Neurophysiology, 55, 1369-1381.

Horstmann, G.A., \& Dietz, V. (1990). A basic posture control mechanism: The stabilization of the centre of gravity. Electroencephalography and Clinical Neurophysiology, 76, 165-176.

Kerwin, D.G., \& Trewartha, G. (2001). Strategies for maintaining a handstand in the anteriorposterior direction. Medicine \& Science in Sports \& Exercise, 33, 1182-1188.

Kuo, A.D. (1995). An optimal control model for analyzing human postural balance. IEEE Transactions on Biomedical Engineering, 42, 87-101.

Nashner, L.M. (1976). Adapting reflexes controlling the human posture. Experimental Brain Research, 26, 59-72.

Nashner, L.M. (1979). Organization and programming of motor activity during posture control. In R. Granit \& O. Pompeiano (Eds.) Progress in brain research, Volume 50, reflex control of posture and movement (pp. 177-184). Amsterdam: Elsevier/NorthHolland Biomedical Press.

Schumann, T., Redfern, M.S., Furman, J.M., El-Jaroudi, A., \& Chaparro, L.F. (1995). Timefrequency analysis of postural sway. Journal of Biomechanics, 28, 603-607.

Slobounov, S.M., \& Newell, K.M. (1996). Postural dynamics in upright and inverted stances. Journal of Applied Biomechanics, 12, 185-196.

Soames, R.W., \& Atha, J. (1982). The spectral characteristics of postural sway behaviour. European Journal of Applied Physiology, 49, 169-177.

Winter, D.A., Patla, A.E., \& Frank, J.S. (1990). Assessment of balance control in humans. Medical Progress through Technology, 16, 31-51.

Winter, D.A., Patla, A., \& Prince, F. (1996). The inverted pendulum of balance control in standing: Control interpretations based on Fourier analysis. In Proceedings of the Canadian Society of Biomechanics, $9^{\text {th }}$ Biennial Conference, Vancouver. 
Wood, G.A., \& Jennings, L.S. (1979). On the use of spline functions for data smoothing. Journal of Biomechanics, 12, 477-479.

Wu, G., \& Zhao, W. (1997). The role of mechanoreceptive information in the stability of human upright posture: A theoretical consideration. Motor Control, 1, 3-19.

Yang, J.F., Winter, D.A. \& Wells, R.P. (1990). Postural dynamics in the standing human. Biological Cybernetics, 62, 309-320.

Yeadon, M.R. (1990a). The simulation of aerial movement. Part I: The determination of orientation angles from film data. Journal of Biomechanics, 23, 59-66.

Yeadon, M.R. (1990b). The simulation of aerial movement. Part II: A mathematical inertia model of the human body. Journal of Biomechanics, 23, 67-74.

Yeadon, M.R., \& Mikulcik, E.C. (1996). The control of non-twisting somersaults using configuration changes. Journal of Biomechanics, 29, 1341-1348.

Zatsiorsky, V.M., \& Duarte, M. (2000). Rambling and trembling in quiet standing. Motor Control, 4, 185-200.

\section{Appendix: Inverse Dynamics Equations}

\section{Nomenclature}

$H$ : hand segment

A: arm segment

$B$ : body (trunk and head) segment

$C$ : leg segment

$J_{1}$ : wrist joint

$J_{2}$ : shoulder joint

$J_{3}$ : hip joint

$\left(x_{\mathrm{i}}, z_{\mathrm{i}}\right)$ : joint center coordinates

$\left(x_{\mathrm{j}}, z_{\mathrm{j}}\right)$ : segment mass center coordinates

$\left(x_{\mathrm{p}}, z_{\mathrm{p}}\right)$ : point $P$ of force application $\left(z_{\mathrm{p}}\right.$ is assumed $\left.=0\right)$

$\ddot{x}_{\mathrm{j}}$ : horizontal linear accelerations of segment mass centers

$\dddot{z}_{\mathrm{j}}$ : vertical linear accelerations of segment mass centers

$I$ : moment of inertia about segment mass centers

$\ddot{\phi}_{\mathrm{j}}$ : segment angular accelerations

$(i=1,3) ;(j=\mathrm{h}, \mathrm{a}, \mathrm{b}$, or $\mathrm{c})$

Resolving horizontally and vertically (Newton's Second Law) and taking moments about the mass center (torque is the rate of change of angular momentum) for each of the four segments $(H, A, B, C)$ results in 12 equations for six joint reaction forces, three torques, and three angular accelerations. Eliminating the joint reaction forces results in six equations for $T_{1}, T_{2}, T_{3}, \ddot{\phi}_{\mathrm{a}}, \ddot{\phi}_{\mathrm{b}}, \ddot{\phi}_{\mathrm{c}}$. Alternatively, these six equations may be derived directly as follows:

Resolving vertically for the whole system $(H, A, B, C)$ gives:

$$
R-m g=m_{\mathrm{a}} \ddot{z}_{\mathrm{a}}+m_{\mathrm{b}} \ddot{z}_{\mathrm{b}}+m_{\mathrm{c}} \ddot{z}_{\mathrm{c}}
$$

Resolving horizontally for the whole system $(H, A, B, C)$ gives:

$$
F=m_{\mathrm{a}} \ddot{x}_{\mathrm{a}}+m_{\mathrm{b}} \ddot{x}_{\mathrm{b}}+m_{\mathrm{c}} \ddot{x}_{\mathrm{c}}
$$

Taking moments about $J_{1}$ for $H$ gives:

$$
-T_{1}+F\left(z_{1}-z_{\mathrm{p}}\right)+R\left(x_{\mathrm{p}}-x_{1}\right)-m_{\mathrm{h}} g\left(x_{\mathrm{h}}-x_{1}\right)=0
$$


Taking moments about $J_{2}$ for $H$ and $A$ gives:

$$
\begin{gathered}
-T_{2}+F\left(z_{2}-z_{\mathrm{p}}\right)-R\left(x_{2}-x_{\mathrm{p}}\right)+m_{\mathrm{h}} g\left(x_{2}-x_{\mathrm{h}}\right)+m_{\mathrm{a}} g\left(x_{2}-x_{\mathrm{a}}\right)= \\
I_{\mathrm{a}} \dot{\phi}_{\mathrm{a}}+m_{\mathrm{a}} \ddot{x}_{\mathrm{a}}\left(z_{2}-z_{\mathrm{a}}\right)-m_{\mathrm{a}} \ddot{\mathrm{z}}_{\mathrm{a}}\left(x_{2}-x_{\mathrm{a}}\right)
\end{gathered}
$$

Taking moments about $J_{3}$ for $H, A$, and $B$ gives:

$$
\begin{gathered}
-T_{3}+F\left(z_{3}-z_{\mathrm{p}}\right)-R\left(x_{3}-x_{\mathrm{p}}\right)+ \\
m_{\mathrm{h}} g\left(x_{3}-x_{\mathrm{h}}\right)+m_{\mathrm{a}} g\left(x_{3}-x_{\mathrm{a}}\right)+m_{\mathrm{b}} g\left(x_{3}-x_{\mathrm{b}}\right)= \\
I_{\mathrm{a}} \ddot{\phi}_{\mathrm{a}}+I_{\mathrm{b}} \ddot{\phi}_{\mathrm{b}}+m_{\mathrm{a}} \ddot{x}_{\mathrm{a}}\left(z_{3}-z_{\mathrm{a}}\right)+m_{\mathrm{b}} \ddot{x}_{\mathrm{b}}\left(z_{3}-z_{\mathrm{b}}\right)-m_{\mathrm{a}} \ddot{z}_{\mathrm{a}}\left(x_{3}-x_{\mathrm{a}}\right)-m_{\mathrm{b}} \ddot{z}_{\mathrm{b}}\left(x_{3}-x_{\mathrm{b}}\right)
\end{gathered}
$$

Taking moments about $P$ for the whole system gives:

$$
\begin{gathered}
-m g\left(x-x_{\mathrm{p}}\right)=I_{\mathrm{a}} \ddot{\phi}_{\mathrm{a}}+I_{\mathrm{b}} \ddot{\phi}_{\mathrm{b}}+I_{\mathrm{c}} \ddot{\phi}_{\mathrm{c}}-m_{\mathrm{a}} \ddot{x}_{\mathrm{a}}\left(z_{\mathrm{a}}-z_{\mathrm{p}}\right)-m_{\mathrm{b}} \ddot{x}_{\mathrm{b}}\left(z_{\mathrm{b}}-z_{\mathrm{p}}\right)- \\
m_{\mathrm{c}} \ddot{x}_{\mathrm{c}}\left(z_{\mathrm{c}}-z_{\mathrm{p}}\right)+m_{\mathrm{a}} \ddot{z}_{\mathrm{a}}\left(x_{\mathrm{a}}-x_{\mathrm{p}}\right)+m_{\mathrm{b}} \ddot{z}_{\mathrm{b}}\left(x_{\mathrm{b}}-x_{\mathrm{p}}\right)+m_{\mathrm{c}} \ddot{z}_{\mathrm{c}}\left(x_{\mathrm{c}}-x_{\mathrm{p}}\right)
\end{gathered}
$$

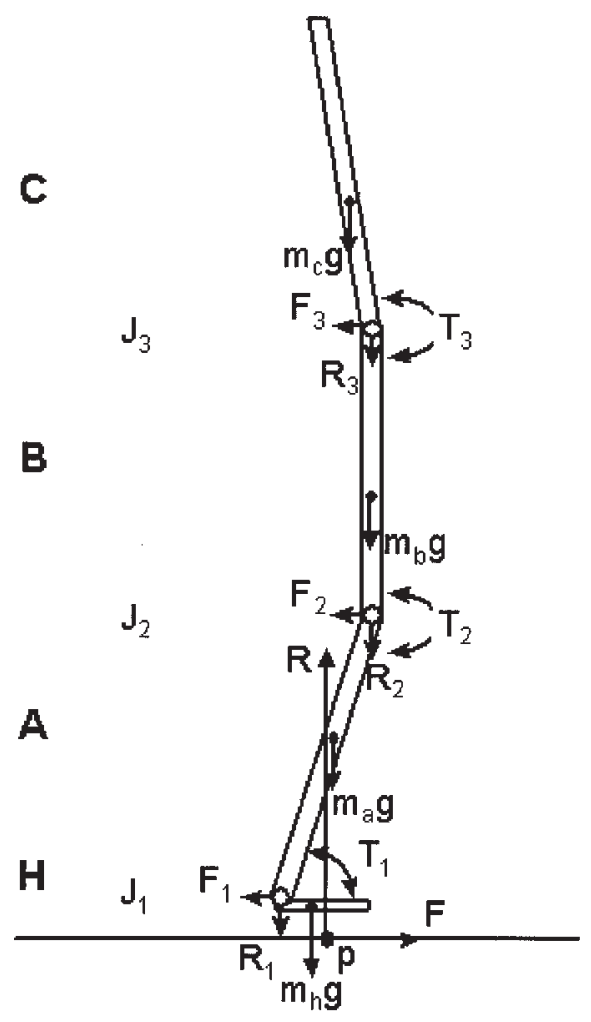

Figure 9 - The four-segment model. 
Using the representation below, trigonometric equivalents of segment mass center linear accelerations can be obtained by differentiating the position values twice.

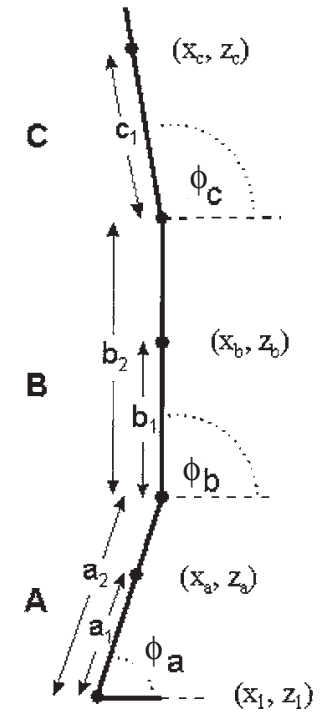

$$
\begin{array}{ll}
x_{\mathrm{a}}=x_{1}+a_{1} \cos \phi_{\mathrm{a}} & \ddot{x}_{\mathrm{a}}=-a_{1} \sin \phi_{\mathrm{a}} \ddot{\phi}_{\mathrm{a}}-a_{1} \cos \phi_{\mathrm{a}} \dot{\phi}_{\mathrm{a}}^{2} \\
z_{\mathrm{a}}=z_{1}+a_{1} \sin \phi_{\mathrm{a}} & \ddot{z}_{\mathrm{a}}=-a_{1} \cos \phi_{\mathrm{a}} \ddot{\phi}_{\mathrm{a}}-a_{1} \sin \phi_{\mathrm{a}} \dot{\phi}_{\mathrm{a}}^{2} \\
x_{\mathrm{b}}=x_{1}+a_{2} \cos \phi_{\mathrm{a}}+b_{1} \cos \phi_{\mathrm{b}} & \ddot{x}_{\mathrm{b}}=-a_{2} \sin \phi_{\mathrm{a}} \ddot{\phi}_{\mathrm{a}}-a_{2} \cos \phi_{\mathrm{a}} \dot{\phi}_{\mathrm{a}}{ }^{2}- \\
& b_{1} \sin \phi_{\mathrm{b}} \ddot{\phi}_{\mathrm{b}}-b_{1} \cos \phi_{\mathrm{b}} \dot{\phi}_{\mathrm{b}}{ }^{2} \\
z_{\mathrm{b}}=z_{1}+a_{2} \sin \phi_{\mathrm{a}}+b_{1} \sin \phi_{\mathrm{b}} & \ddot{z}_{\mathrm{b}}=a_{2} \cos \ddot{\mathrm{d}}_{\mathrm{a}}-a_{2} \sin \phi_{\mathrm{a}} \dot{\phi}_{\mathrm{a}}{ }^{2}+ \\
& b_{1} \cos \ddot{\mathrm{\phi}}_{\mathrm{b}}-b_{1} \sin \phi_{\mathrm{b}} \dot{\phi}_{\mathrm{b}}{ }^{2}
\end{array}
$$$$
x_{\mathrm{c}}=x_{1}+a_{2} \cos \phi_{\mathrm{a}}+b_{2} \cos \phi b+c_{1} \cos \phi_{\mathrm{c}} \quad \ddot{x}_{\mathrm{c}}=-a_{2} \sin \phi_{\mathrm{a}} \ddot{\phi}_{\mathrm{a}}-a_{2} \cos \phi_{\mathrm{a}} \dot{\phi}_{\mathrm{a}}^{2}-
$$$$
b_{2} \sin \phi_{\mathrm{b}} \ddot{\phi}_{\mathrm{b}}-b_{2} \cos \phi_{\mathrm{b}} \dot{\phi}_{\mathrm{b}}^{2}-c_{1} \sin \phi_{\mathrm{c}} \ddot{\phi}_{\mathrm{c}}-
$$$$
c_{1} \cos \phi_{\mathrm{c}} \dot{\phi}_{\mathrm{c}}^{2}
$$$$
z_{\mathrm{c}}=z_{1}+a_{2} \sin \phi_{a}+b_{2} \sin \phi_{b}+c_{1} \sin \phi_{\mathrm{c}} \quad \ddot{z}_{\mathrm{c}}=a_{2} \cos \phi_{\mathrm{a}} \ddot{\phi}_{\mathrm{a}}-a_{2} \sin \phi_{\mathrm{a}} \dot{\phi}_{\mathrm{a}}^{2}+
$$$$
b_{2} \cos \phi_{\mathrm{b}} \ddot{\phi}_{\mathrm{b}}-b_{2} \sin \phi_{\mathrm{b}} \dot{\phi}_{\mathrm{b}}^{2}+c_{1} \cos \phi_{\mathrm{c}} \ddot{\phi}_{\mathrm{c}}-
$$$$
c_{1} \sin \phi_{c} \dot{\phi}_{c}^{2}
$$

By substituting the trigonometric equivalents in place of the linear acceleration terms in equations (A1)-(A6) and rearranging terms, we obtain six linear equations in the following form to solve for the six unknowns $T_{1}, T_{2}, T_{3}, \dot{\phi}_{\mathrm{a}}, \ddot{\phi}_{\mathrm{b}}, \ddot{\phi}_{\mathrm{c}}$ 


$$
\begin{aligned}
& A_{11} T_{1}+A_{12} T_{2}+A_{13} T_{3}+A_{14} \ddot{\phi}_{\mathrm{a}}+A_{15} \ddot{\phi}_{\mathrm{b}}+A_{16} \ddot{\phi}_{\mathrm{c}}=B_{1} \\
& A_{21} T_{1}+A_{22} T_{2}+A_{23} T_{3}+A_{24} \ddot{\phi}_{\mathrm{a}}+A_{25} \ddot{\phi}_{\mathrm{b}}+A_{26} \ddot{\phi}_{\mathrm{c}}=B_{2} \\
& A_{31} T_{1}+A_{32} T_{2}+A_{33} T_{3}+A_{34} \ddot{\phi}_{\mathrm{a}}+A_{35} \ddot{\phi}_{\mathrm{b}}+A_{36} \ddot{\phi}_{\mathrm{c}}=B_{3} \\
& A_{41} T_{1}+A_{42} T_{2}+A_{43} T_{3}+A_{44} \ddot{\phi}_{\mathrm{a}}+A_{45} \ddot{\phi}_{\mathrm{b}}+A_{46} \ddot{\phi}_{\mathrm{c}}=B_{4} \\
& A_{51} T_{1}+A_{52} T_{2}+A_{53} T_{3}+A_{54} \ddot{\phi}_{\mathrm{a}}+A_{55} \ddot{\phi}_{\mathrm{b}}+A_{56} \ddot{\phi}_{\mathrm{c}}=B_{5} \\
& A_{61} T_{1}+A_{62} T_{2}+A_{63} T_{3}+A_{64} \ddot{\phi}_{\mathrm{a}}+A_{65} \ddot{\phi}_{\mathrm{b}}+A_{66} \ddot{\phi}_{\mathrm{c}}=B_{6}
\end{aligned}
$$

All of the terms held in the coefficients $A_{11}$ through $B_{6}$ can be derived from video or force data at each instant in time. A linear equation solver is used to determine estimates for the six unknowns at each time instant.

However, a number of the equation coefficients involve $\cos \phi_{\mathrm{a}}, \cos \phi_{\mathrm{b}}, \cos \phi_{\mathrm{c}}$, which result in singularities in the calculated torques and angular accelerations around $\phi=90^{\circ}(j=\mathrm{a}, \mathrm{b}, \mathrm{c})$. To avoid this problem, three more equations are added, using video estimates $e_{1}, e_{2}, e_{3}$ of the angular accelerations $\ddot{\phi}_{\mathrm{a}}, \ddot{\phi}_{\mathrm{b}}, \ddot{\phi}_{\mathrm{c}}$. These may be written as:

$$
\begin{aligned}
& A_{44} \ddot{\phi}_{\mathrm{a}}=A_{44} e_{1} \\
& A_{55} \ddot{\phi}_{\mathrm{b}}=A_{55} e_{2} \\
& A_{66} \ddot{\phi}_{\mathrm{c}}=A_{66} e_{3}
\end{aligned}
$$

which matches the coefficients of $\ddot{\phi}_{a}, \ddot{\phi}_{b}, \ddot{\phi}_{c}$ in the last three of the six previous equations. This gives an over-determined system of nine equations for the six unknowns, and a least-squares equation solver results in solutions without singularities. 